\title{
riccafd
}

Revista Iberoamericana de Ciencias de la Actividad Física y el Deporte

\section{EVALUACIÓN DE LOS CENTROS LATERALES EN CONTRA Y SAQUES DE BANDA A FAVOR EN FÚTBOL SEMIPROFESIONAL. ESTUDIO DE CASO}

\section{EVALUATION OF THE LATERAL CENTRES AGAINST AND THROW-INS IN FAVOUR IN SEMI-PROFESSIONAL FOOTBALL. CASE STUDY}

\author{
Álvaro Jesús García-Paúl' ${ }^{1}$, Eduardo Valera-Sánchez ${ }^{1}$ y Alfonso Castillo-Rodríguez² \\ ${ }^{1}$ Facultad de Ciencias del Deporte, Universidad de Granada, Granada, España. \\ 2 Departamento de Educación Física y Deportiva, Universidad de Granada, Granada, España.
}

Autor de correspondencia: Alfonso Castillo-Rodríguez. Facultad de Ciencias del Deporte, Universidad de Granada. Ctra. Alfacar s/n, 18011 - Granada (España). acastillo@ugr.es +34620397897

DOI: http://dx.doi.org/10.24310/riccafd.2019.v8i1.5767

\section{RESUMEN}

El scouting deportivo, match analysis, o análisis del juego en deportes de interacción ha cobrado especial interés desde la última década. La preocupación y la importancia en el fútbol por la victoria hacen que se sume la forma de juego y las fortalezas que posee el equipo que te vas a enfrentar. Para ello, esta nueva metodología de análisis, evaluación y valoración de acciones del juego permiten obtener información muy pertinente por parte de los entrenadores. El objetivo de este estudio fue analizar a través de la metodología observacional, las acciones erróneas y correctas que se llevan a cabo en un equipo semiprofesional de fútbol en partidos reales, concretamente en los centros laterales en contra y los saques de banda a favor en fútbol. Veintitres jugadores semiprofesionales del Sur de España, fueron los participantes del estudio. Se crearon instrumentos ad-hoc para el acotamiento de las acciones a evaluar, siendo el número total en cada uno de los problemas a analizar de 50. Los resultados mostraron detalles de las categorías y elementos que determina un problema real en el equipo analizado, permitiendo un margen de mejora con los aspectos reseñados como positivos. Como conclusión, los principales hallazgos de este estudio mostraron que el equipo analizado de fútbol semiprofesional posee problemas en el juego como son en los saques de banda a favor y en los centros laterales defensivos. Esto ha sido hallado gracias a la metodología utilizada, que ha podido de manera 
objetiva analizar y valorar acciones que se dan en los partidos de competición, ayudando de esta manera al entrenador para realizar tareas enfocadas a paliar y mejorar el rendimiento de sus jugadores, optimizando el aspecto táctico del equipo, objetivo fundamental de la metodología activa.

Palabras clave: competición, metodología observacional, fútbol, rendimiento, táctica.

\section{SUMMARY}

Sports scouting, match analysis, or game analysis in interaction sports has gained special interest since the last decade. The concern and importance in football for the victory makes it add to the form of game and the strengths that the team that you are going to face has. For this, this new methodology of analysis, evaluation and assessment of game actions allow obtaining very pertinent information from the coaches. The objective of this study was to analyze, through the observational methodology, the erroneous and correct actions that are carried out in a semi-professional soccer team in real matches, specifically in the lateral centers against and the throw-ins in football. . Twenty-three semi-professional players from the South of Spain, were the participants of the study. Ad-hoc instruments were created for the delimitation of the actions to be evaluated, with the total number in each of the problems to be analyzed of 50 . The results showed details of the categories and elements that determine a real problem in the analyzed equipment, allowing a margin of improvement with the aspects outlined as positive. In conclusion, the main findings of this study showed that the analyzed team of semi-professional soccer has problems in the game as they are in throw-ins in favor and defensive lateral centers. This has been found thanks to the methodology used, which has been able to objectively analyze and assess actions that occur in the competition matches, thus helping the coach to perform tasks focused on alleviating and improving the performance of their players, optimizing the tactical aspect of the team, fundamental objective of the active methodology.

Key words: competition, observational methodology, soccer, performance, tactics.

\section{INTRODUCCIÓN}

El deporte como sistema social tiene que avanzar de la mano de las nuevas tecnologías, es decir, debe responder a las necesidades en los diferentes ámbitos del deporte, para ello debemos introducir elementos informáticos que amplían y perfeccionan enormemente sus posibilidades de seguir desarrollándose (Brodie \& Thornhill, 1983). En los últimos años el deporte se ha aprovechado de los avances tecnológicos incorporándolos a sus estructuras de trabajo. Debido a esto, actualmente se disponen de sistemas sofisticados de registro de la actividad deportiva que desarrollan diferentes ámbitos. (Jonsson, Blanco, Losada, \& Anguera, 2004). 
Desde el punto de vista que ofrece la metodología observacional, en todos los deportes de equipo, y específicamente en el fútbol, nos permiten una gran gama de posibilidades de estudio. Existen multitud de aspectos cuya importancia podemos suponer desde una perspectiva de técnico, o desde un punto de vista descriptivo, o incluso de aficionado. Pero no resulta fácil plantear el andamiaje metodológico que permita investigar acerca de la multitud de relaciones existentes de forma consistente. La investigación sobre cualquier modalidad de actividad física y de deporte te impone una disciplina al estudioso, y exige el cumplimiento de unos condicionantes y requisitos (Anguera, 2004).

Todos los profesionales del fútbol (entrenadores, preparadores físicos, psicólogos, entre otros), tanto en el ámbito de la formación como en la alta competición, tienen una preocupación común: la de hacer más eficientes los procesos de enseñanza-aprendizaje y entrenamiento. Esto hizo que pudiésemos ver en las últimas décadas a un desarrollo significativo de procedimientos, métodos, sistemas y técnicas de desarrollo del entrenamiento deportivo que tanto contribuyeron para la evolución de la modalidad. Es por ello que entendemos que la metodología observacional en general y las técnicas de análisis secuencial y de coordenadas polares en particular se presentan como un modelo de análisis pertinente e innovador que puede ayudar a percibir mejor las relaciones, las conductas y los comportamientos de jugadores y equipos en el fútbol (Silva, Sánchez Bañuelos, Garganta, \& Anguera, 2005).

La Metodología Observacional (MO) ha ido desarrollándose de manera notoria en estos últimos años, y se puede observar en publicaciones españolas (Anguera, 2010; Anguera, Blanco-Villaseñor, Losada, \& Hernández-Mendo, 2000) y extranjeras (Anguera, 2003; Bakeman \& Quera, 2011; Sánchez-Algarra \& Anguera, 2013; Thompson, Felce, \& Symons, 2000; Yoder \& Simmons, 2010). El análisis tradicional en el fútbol se ha centrado en la mayoría de las ocasiones en el uso de análisis estadísticos y representaciones gráficas que aportan información sobre la frecuencia, distribución espacial de los pases de los jugadores, tiros a puerta, o estilos de defensa por nombrar algunos de los ejemplos. Pero el rendimiento de los jugadores resulta de estrategias y tácticas que corresponderían con los patrones de conducta que se dan repetidamente a lo largo de un partido (Anguera, 2004).

El análisis de los partidos de fútbol es uno de los aspectos que está adquiriendo mucha importancia en este deporte (Carling, Williams, \& Reilly, 2005). Cada día con mayor frecuencia, los miembros del cuerpo técnico de un equipo de fútbol dedican mayores recursos, esfuerzos y tiempo en analizar a los equipos tanto el propio equipo como el equipo rival). Uno de los objetivos inmediatos del análisis de partidos es identificar las fortalezas y debilidades del juego de los rivales (Carling, Bloomfield, Nelsen, \& Reilly, 2008) con los que se enfrentarán los equipos. A más largo plazo, los investigadores y equipos técnicos analizan los modelos de juego de los equipos que tienen éxito (Robles y Castellano, 2012). Sin embargo, debemos tener cuidado con el uso de algunos criterios que, al presentar valores por debajo de los deseados, deben 
ser mejorados para poder continuar con las sucesivas fases de la investigación sistematizada (Anguera et al., 2000).

El objetivo del presente estudio es analizar a través de la metodología observacional, las acciones erróneas y correctas que se llevan a cabo en un equipo semiprofesional de fútbol en partidos reales, concretamente en los centros laterales en contra y los saques de banda a favor en fútbol.

\section{MATERIAL Y MÉTODO}

\section{Participantes}

La población elegida para realizar esta investigación han sido 23 futbolistas semiprofesionales de un equipo de fútbol de segunda división B del grupo IV. Los casos estudiados no los podemos considerar representativos de toda la población de futbolistas semiprofesionales, y por lo tanto, generalizar los resultados obtenidos, sólo se podrían aplicar a casos similares (Angera, 1983).

\section{Instrumentos}

La valoración llevada a cabo en los partidos de competición fue realizada a través del Nac Sport® (NAC Sport S.L., España). La observación fue directa después de los partidos de competición llevados a cabo por el equipo para la observación de los 2 problemas planteados (en los centros laterales en contra y los saques de banda a favor).

\section{Procedimiento}

El método utilizado para este trabajo ha sido en primer lugar, utilizar una hoja de Microsoft Excel para anotar las variables que se deben cumplir en cada acción táctica analizada. En este caso, se analizaron 50 acciones por cada problema planteado, que se dieron entre 3 y 4 partidos cada problema. Para el análisis de video se utilizó el programa informático Nac Sport®, en el que se crearon las categorías y elementos que se deseaban registrar para valorar los aciertos y errores del equipo analizado.

Una vez terminado el análisis, se extrajeron los resultados a modo de porcentajes y frecuencias con las que se cumplen o no las categorías propuestas y los elementos que los subyacen. Finalmente, se llevará a cabo unas tareas con una metodología novedosa y planteando un problema para que los futbolistas busquen varias soluciones del mismo.

En este caso, los problemas planteados tienen aspectos diferenciados y pertenecen a diferentes fases del juego, ya que uno de ellos, implica la posesión del balón, y el otro, implica aspectos relacionados con la táctica en defensa. El primer problema los podemos incluir en ambas fases de ataque, es decir, en el 
ataque organizado según el modelo de juego de nuestro equipo, o si la defensa del equipo rival no está organizada a partir de esta acción también podemos comenzar un contraataque. El segundo problema lo podríamos enmarcar dentro la fase defensiva de nuestro equipo, ya sea con defensa organizada, o con un repliegue o pressing tras un intento de contra del equipo rival. Las categorías y elementos dependen unos de otros por lo que se pueden considerar que no son mutuamente excluyentes (Tabla 1).

Tabla 1. Categorías y elementos planteados para la evaluación y valoración de los 2 problemas planteados

\begin{tabular}{|c|c|}
\hline Problema 1: Saques de banda a favor & $\begin{array}{c}\text { Problema 2: Centros laterales } \\
\text { defensivos }\end{array}$ \\
\hline $\begin{array}{l}\text { A. Desenlace de la acción: } \\
\text { 1. Pérdida } \\
\text { 2. Mantenimiento de la posición } \\
\text { B. Quien pierde la posesión: } \\
\text { 1. Lanzador del saque de banda } \\
\text { 2. Otro jugador } \\
\text { C. Como se produce la pérdida: } \\
\text { 1. Balón largo } \\
\text { 2. Pase en corto } \\
\text { 3. Conducción } \\
\text { 4. Tras control } \\
\text { 5. Saque de banda directamente } \\
\text { D. momento de la perdida: } \\
\text { 1. } 0 \text { pases } \\
\text { 2. } 1 \text { o } 2 \text { pases } \\
\text { 3. } 3 \text { o más pases } \\
\text { E. Pase de seguridad: } \\
\text { 1. Si } \\
\text { 2. No } \\
\text { F. Dirección del saque de la pérdida: } \\
\text { 1. atrás } \\
\text { 2. horizontal } \\
\text { 3. adelante } \\
\text { G. Zona perdida: } \\
\text { 1. } 1 \\
\text { 2. } 2 \\
\text { 3. } 3 \\
\text { 4. } 4 \\
\text { H. la continuidad se produce por continuidad: } \\
\text { 1. Tras balón directo } \\
\text { 2. Tras pase en corto } \\
\text { 3. Tras conducción de balón } \\
\text { I. Continuidad: } \\
\text { 1. Tras pase de seguridad } \\
\text { 2. Sin pase de seguridad } \\
\text { J. Dirección del saque que propicia la } \\
\text { continuidad: } \\
\text { 1. Hacia atrás } \\
\text { 2. En horizontal } \\
\text { 3. Hacia delante } \\
\text { K. Zona de inicio donde se produce la } \\
\text { continuidad: } \\
\text { 1. } 1 \\
\text { 2. } 2 \\
\text { 3. } 3 \\
\text { 4. } 4\end{array}$ & $\begin{array}{l}\text { A. Acción posterior del centro lateral: } \\
\text { 1. Remate del equipo rival } \\
\text { 2. Despeje } \\
\text { 3. Parada del portero } \\
\text { 4. Centros que no encuentran a nadie } \\
\text { B. Remate rival: } \\
\text { 1. A portería } \\
\text { 2. Fuera de los } 3 \text { palos } \\
\text { 3. Segundas jugadas } \\
\text { C. Remate del rival a portería: } \\
\text { 1. Gol } \\
\text { 2. Intercepta el defensa } \\
\text { 3. Parada del portero } \\
\text { D. Orientación oponente directo del } \\
\text { rematador: } \\
\text { 1. Buena orientación } \\
\text { 2. Mala orientación } \\
\text { E. Dirección del despeje: } \\
\text { 1. Hacia nuestra propia portería } \\
\text { 2. Hacia la portería rival } \\
\text { 3. Hacia la banda } \\
\text { F. Acción que se produce después del } \\
\text { despeje: } \\
\text { 1. Mantenimiento de la posesión } \\
\text { 2. Córner rival } \\
\text { 3. Saque de banda rival } \\
\text { 4. Segundo despeje } \\
\text { 5. segunda jugada para el equipo rival } \\
\text { G. Zona a la que se dirige el centro } \\
\text { (siempre zona 1): } \\
\text { 1. 1.A } \\
\text { 2. 1.B } \\
\text { 3. 1.C } \\
\text { 4. 1.D }\end{array}$ \\
\hline
\end{tabular}




\section{Análisis Estadístico}

Se han realizado análisis descriptivos y se han llevado a cabo figuras en el software Microsoft Office Excel 2010.

\section{RESULTADOS}

Acerca de los saques de banda a favor, en la figura 1A podemos observar que el número de pérdidas de balón tras un saque de banda a favor es bastante alto, concretamente un $64 \%$ de las veces, y de estas pérdidas, casi en el $80 \%$ de las ocasiones, la posesión se pierde en la acción posterior al saque, es decir, no directamente en el mismo saque, por lo que se presupone que las decisiones posteriores al saque es la causa de la pérdida de la posesión del balón. En la figura $1 \mathrm{C}$, se observa que el $78 \%$ de las pérdidas provienen de acciones técnicas como pase largo, pase corto, conducción y tras control. En la figura 1D se aprecia que el $56 \%$ de las acciones se pierde tras el primer o segundo pase, por lo que, normalmente se podría intuir que el jugador que recibe el saque de banda suele tomar una decisión errónea, concluyendo en la pérdida de balón. Además, casi en el $70 \%$ de las acciones que se pierde el balón, no existe un pase de seguridad para intentar evitar la presión (figura 1E). En la figura 1F, se aprecia que en el $81,25 \%$ de las pérdidas producidas, ha sido en la dirección hacia delante, mientras que los valores de los saques en horizontal y hacia atrás con prácticamente nulos, por lo que tendremos que tener esta situación muy en cuenta a la hora de elaborar tareas para solucionar este problema. Sobre la zona, la pérdida no se produce en una zona aislada, sino que el equipo tiene muchos problemas en todas las zonas del campo, aunque donde menos pérdidas tiene es en zona 1, probablemente porque no todos los equipos ejercen la presión en esta zona, y donde más perdidas se producen es en la última zona ya que hay menos espacios y el equipo defensor suele estar muy junto (figura 1G). Acerca de la continuidad en el juego, se observa que el $66 \%$ de las ocasiones que mantenemos la posesión del balón, se ha producido gracias a pases cortos, por lo que debemos evitar siempre en la medida de lo posible los balones largos y directos tras los saques de banda (figura $1 \mathrm{H}$ ). Finalmente, se extrae en la figura $1 \mathrm{~J}$ que la mayoría de las veces que se produce la continuidad es cuando sacamos hacía delante.

Acerca del segundo problema analizado, los centros laterales defensivos, destacamos la zona a la que se dirige el centro lateral, siendo en su mayoría por las zonas centrales, es decir, las más cercanas a la portería por lo que suelen buscar el remate directo a no ser, que sea muy clara la dejada por parte del rematador. En la figura $2 \mathrm{~A}, 2 \mathrm{~B}$ y $2 \mathrm{C}$ se observan el número de veces que nos rematan por partido y la dirección de los remates. Se aprecia que el $32 \%$ de las acciones son superiores los rivales, teniendo un final negativo para nuestro equipo analizado, que más de la mitad de esas acciones, son dirigidos hacia nuestra propia portería. El dato final (figura 2C) nos indica que el $60 \%$ de las acciones son paradas por nuestro portero, lo que implica un trabajo a realizar en la planificación posterior. Además, se ha analizado que más del $80 \%$ de las acciones, el oponente 


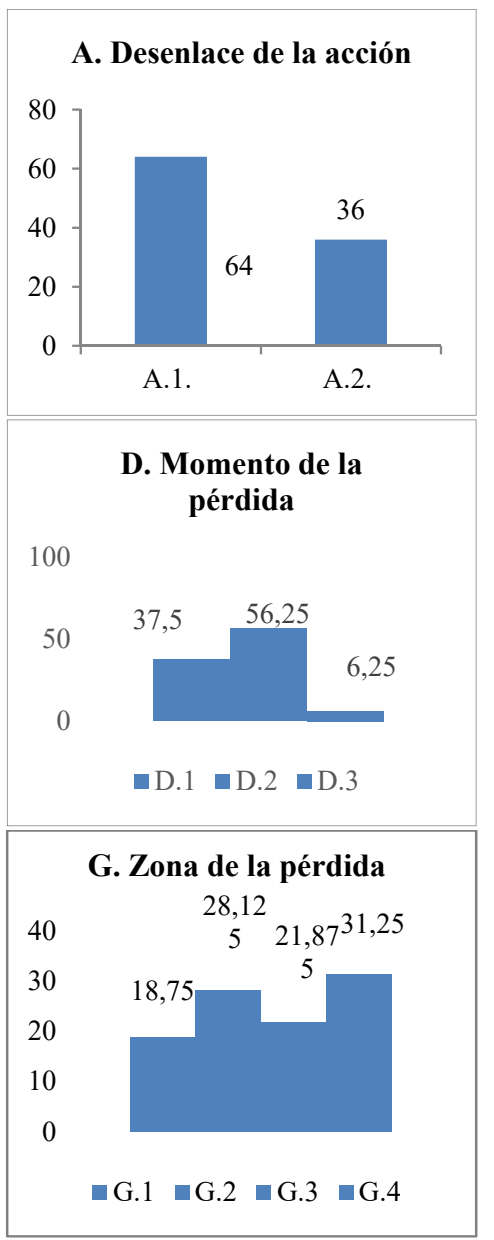

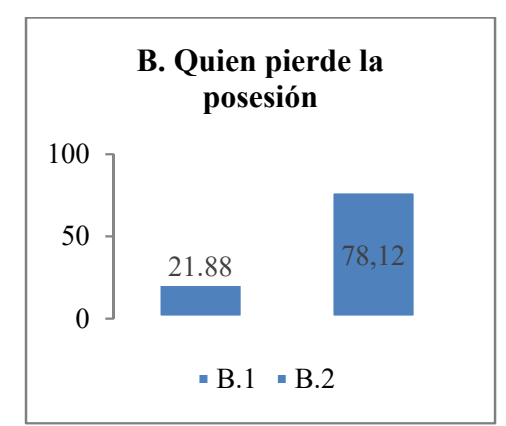

E. Hay pase de seguridad en la pérdida
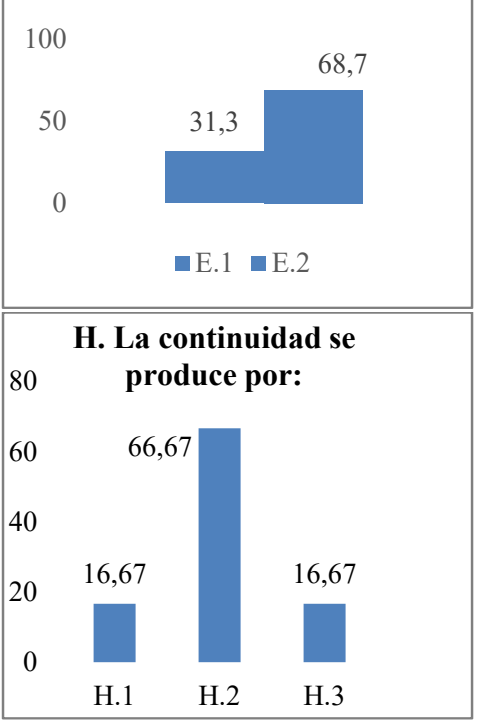

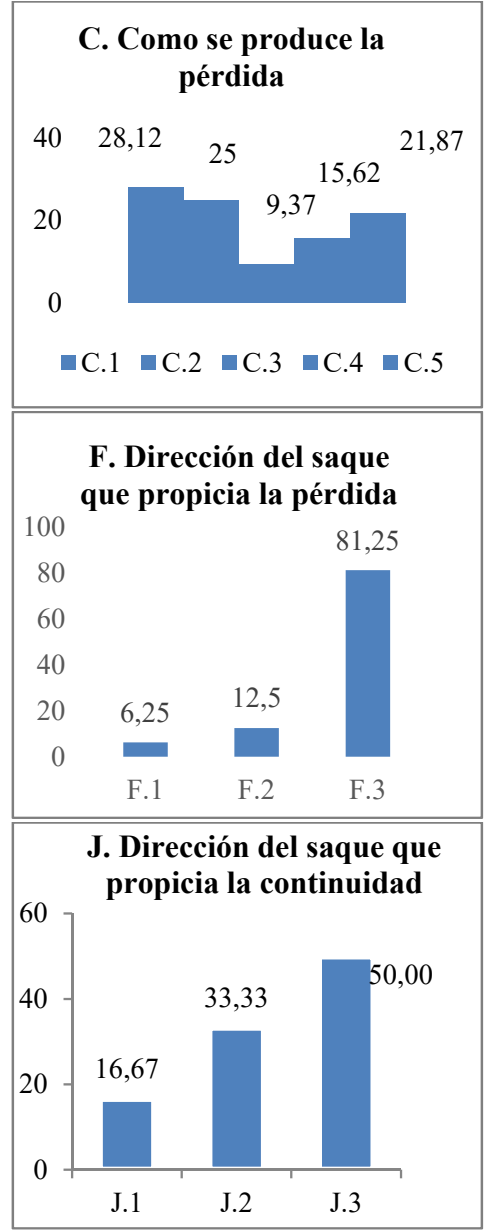

Figura 1. Resultados del análisis de las categorías del problema 1: saques de banda a favor.

de nuestro equipo sobre el rematador se encuentra mal orientado (figura 2D). Finalmente, en la figura 2E se muestra la dirección del despeje, resultando equilibrado entre las zonas y en la $2 \mathrm{~F}$, son acciones producidas después del centro lateral, en el que destaca, que el 30\% termina en córner en contra.

\section{DISCUSIÓN}

El entrenamiento que un deportista de alto nivel tiene como objetivo optimizar sus capacidades o aptitudes, debe ajustarse de manera más parecida posible a la prueba para la que se está preparando desde un enfoque holístico de lo físico y lo psicológico (Bompa, 1993). Por ello, las demandas energéticas de un deporte de equipo son difíciles de cuantificar por diferentes parámetros: multitud de demarcaciones, características de los jugadores, incertidumbre del rival, estado del terreno de juego, condiciones climatológicas (Leo Marcos, Pulido, SánchezOliva, Candela, \& García-Calvo, 2012).

Durante los últimos años, la planificación en los deportes de equipo ha evolucionado de manera significativa en cuanto a formas y métodos de entrenamiento. 


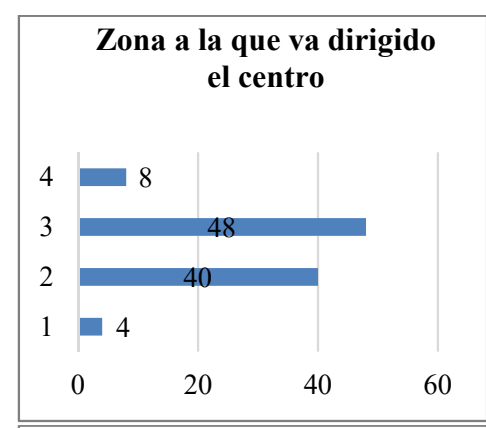

C. Remates del equipo rival a portería

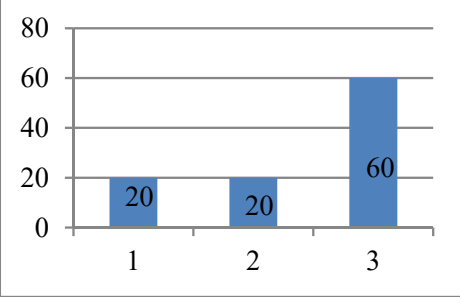

F. Acciones producidas después de despeje

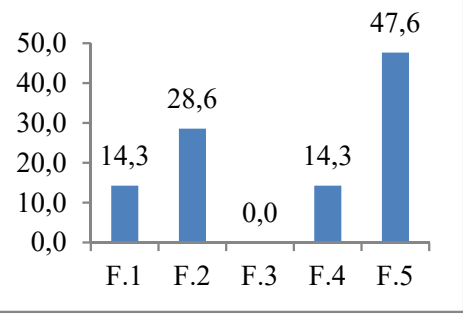

A. Ante centros laterales

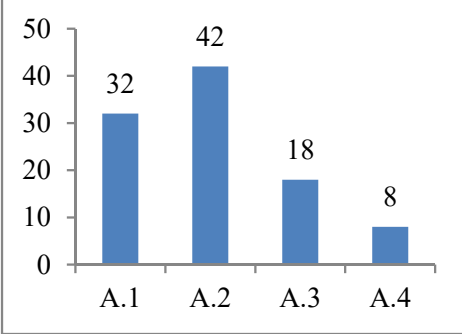

D. Orientación del oponente directo al rematador

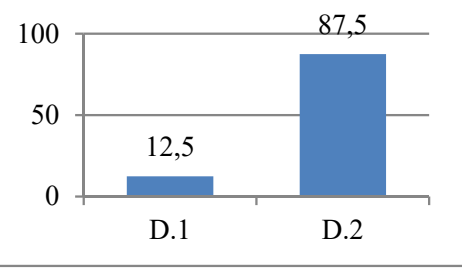

D.1

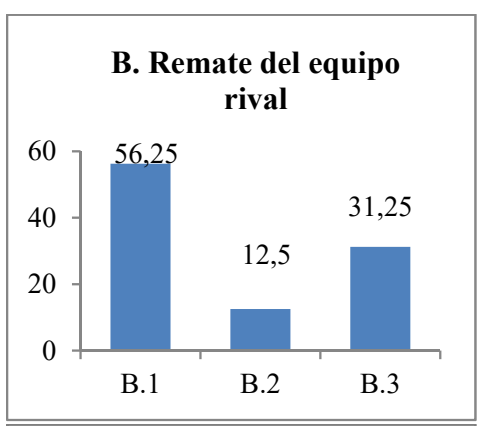

E. Dirección del despeje

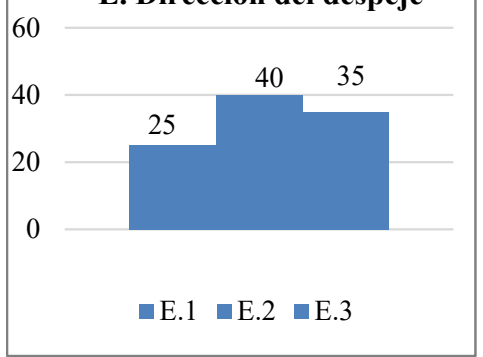

Figura 2. Resultados del análisis de las categorías del problema 2: centros laterales defensivos

Hace unos años, los métodos de trabajo en los deportes de equipo y entre ellos el fútbol, se basaban en líneas generales, en métodos tradicionales que eran utilizados para preparar deportistas que practicaban deportes individuales, tomando como referencia ciencias como las matemáticas o química (Arjol, 2012).

Los juegos reducidos, también conocidos como "acondicionamiento basado en la habilidad" o "entrenamiento basado en el juego" (Gabbet, 2006; Gabbet, Jenkins, \& Abernethy, 2009), son situaciones lúdico-deportivas (Parlebas, 2001), en las que interactúan de forma conjunta todos los elementos del juego de una manera flexible (Wein, 1995). Los juegos reducidos nos ofrecen un estímulo de entrenamiento específico que reproduce las demandas fisiológicas de la competición (Gabbett, 2010).

Los juegos reducidos nos permiten la posibilidad de entrenar el modelo de juego pero tienen ciertas limitaciones, por ejemplo, es casi imposible poder cuantificar la carga del entrenamiento y necesitaríamos de la tecnología, es casi imposible comparar dos jugadores, ya que la forma de jugar de cada jugador determinara la intensidad y la carga que le supone el entrenamiento, por lo que estos juegos reducidos pueden que estimulen los suficiente desde el punto de vista de la condición física a ciertos jugadores y puede que haya otros que no lleguen a este umbral (Casamichana, San Román, Calleja, \& Castellano, 2015). 


\section{CONCLUSIONES}

Los principales hallazgos de este estudio mostraron que el equipo analizado de fútbol semiprofesional posee problemas en el juego como son en los saques de banda a favor y en los centros laterales defensivos. Esto ha sido hallado gracias a la metodología utilizada, que ha podido de manera objetiva analizar y valorar acciones que se dan en los partidos de competición, ayudando de esta manera al entrenador para realizar tareas enfocadas a paliar y mejorar el rendimiento de sus jugadores, optimizando el aspecto táctico del equipo, objetivo fundamental de la metodología activa.

\section{REFERENCIAS BIBLIOGRÁFICAS}

Anguera, M. T. (1983). Manual de técnicas de observación. México: Trillas.

Anguera, M. T., Blanco, A., Losada, J. L., \& Hernández, A. (2000). La metodología observacional en el deporte: conceptos básicos. Lecturas: EF y Deportes. Revista Digital, 24(5), 63-82.

Argilaga, M. T. A. (2004). Hacia la búsqueda de estructuras regulares en la observación del fútbol: detección de patrones temporales. Cultura, ciencia y deporte: revista de ciencias de la actividad física y del deporte de la Universidad Católica de San Antonio, 1, 15-20.

Argilaga, M. T. A., Villaseñor, A. B., Mendo, A. H., \& López, J. L. L. (2011). Diseños observacionales: ajuste y aplicación en psicología del deporte. Cuadernos de Psicología del Deporte, 11(2), 63-76.

Arjol, J. L. (2012). La planificación actual del entrenamiento en fútbol.: Análisis comparado del enfoque estructurado y la periodización táctica. Acción Motriz, 8, 27-37.

Bompa, T. (1993). Periodización de la fuerza. Ed. Biosystem Serv. Educativo.

Brodie, D. A., \& Thornhill, J. J. (1983). Microcomputing in Sport and Physical Education.

Carling, C., Bloomfield, J., Nelsen, L., \& Reilly, T. (2008). The role of motion analysis in elite soccer. Sports Medicine, 38(10), 839-862.

Carling, C., Williams, A. M., \& Reilly, T. (2005). The handbook of soccer match analysis.

Casamichana, D., San Román, J., Calleja, J., \& Castellano, J. (2015). Los juegos reducidos en el entrenamiento del fútbol. Barcelona: Futbol De Libro.

Gabbet, T.; Jenkins, D.; Abernethy, B. (2009). Game-based training for improving skill and physical fitness in team sport athletes. International Journal of Sports Science \& Coaching, 4(2), 273- 283.

Gabbett, T. J. (2010). GPS analysis of elite women's field hockey training and competition. Journal of Strength and Conditioning Research, 24(5), 1321-1324

Hernández Mendo, A., Castellano, J., Camerino, O., Jonsson, G., Villaseñor, Á. B., Lopes, A., \& Anguera, M. T. (2014). Programas informáticos de registro, control de calidad del dato, y análisis de datos. Revista de Psicología del Deporte, 23(1).

Jonsson, G. K., Blanco-Villaseñor, A., Losada, J. L., \& Anguera, M. T. (2004). Avances en la codificación y análisis de eventos deportivos: ilustración empírica en el fútbol. Metodología de las Ciencias del Comportamiento, 2004, 317-322. 
Parlebas, P. (2001). Juegos, deportes y sociedades. Léxico de praxiología motriz. Barcelona: Paidotribo.

Robles, F. J., \& Castellano, J. (2012). Comparación entre el juego ofensivo de la selección española de fútbol y sus rivales en la Eurocopa'08 y Mundial'10. Revista Iberoamericana de Psicología del Ejercicio y el Deporte, 7(2).

Silva, A., Sánchez Bañuelos, F., Garganta, J., \& Anguera, M. T. (2005). Patrones de juego en el fútbol de alto rendimiento. Análisis secuencial del proceso ofensivo en el campeonato del mundo Corea-Japón 2002. Cultura, Ciencia y Deporte, 1(2).

Wein, H. (1995). Fútbol a la medida del niño. Un óptimo modelo de formación como clave de futuros éxitos. Madrid: RFEF. 